\title{
Mimetic dark matter
}

\author{
Ali H. Chamseddine ${ }^{a, b}$ and Viatcheslav Mukhanov ${ }^{c, d, e}$ \\ ${ }^{a}$ Physics Department, American University of Beirut, \\ Beirut, Lebanon \\ ${ }^{b}$ I.H.E.S., \\ F-91440 Bures-sur-Yvette, France \\ ${ }^{c}$ Theoretical Physics, Ludwig Maxmillians University, \\ Theresienstr. 3\%, 80333 Munich, Germany \\ ${ }^{d}$ LPT de l'Ecole Normale Superieure, Chaire Blaise Pascal, \\ 24 rue Lhomond, 75231 Paris cedex, France \\ ${ }^{e}$ MPI for Physics, \\ Foehringer Ring, 6, 80850, Munich, Germany \\ E-mail: chams@aub.edu.1b, viatcheslav.mukhanov@physik.uni-muenchen.de
}

ABSTRACT: We modify Einstein's theory of gravity, isolating the conformal degree of freedom in a covariant way. This is done by introducing a physical metric defined in terms of an auxiliary metric and a scalar field appearing through its first derivatives. The resulting equations of motion split into a traceless equation obtained through variation with respect to the auxiliary metric and an additional differential equation for the trace part. As a result the conformal degree of freedom becomes dynamical even in the absence of matter. We show that this extra degree of freedom can mimic cold dark matter.

KeYwords: Cosmology of Theories beyond the SM, Classical Theories of Gravity ARXIV EPRINT: 1308.5410 
Consider a physical metric $g_{\mu \nu}$ to be a function of a scalar field $\phi$ and an auxiliary metric $\tilde{g}_{\mu \nu}$, defined by

$$
g_{\mu \nu}=\left(\tilde{g}^{\alpha \beta} \partial_{\alpha} \phi \partial_{\beta} \phi\right) \tilde{g}_{\mu \nu} \equiv P \tilde{g}_{\mu \nu} .
$$

It is obvious that in this case the metric $g_{\mu \nu}$ is invariant with respect to the conformal transformation of the auxiliary metric $\tilde{g}_{\mu \nu}$, that is, $g_{\mu \nu} \rightarrow g_{\mu \nu}$ when $\tilde{g}_{\mu \nu} \rightarrow \Omega^{2} \tilde{g}_{\mu \nu}$. The action is constructed in terms of the physical metric $g_{\mu \nu}$, which will be considered as a function of the scalar field $\phi$ and the auxiliary metric $\tilde{g}_{\mu \nu}$, that is,

$$
S=-\frac{1}{2} \int d^{4} x \sqrt{-g\left(\tilde{g}_{\mu \nu}, \phi\right)}\left[R\left(g_{\mu \nu}\left(\tilde{g}_{\mu \nu}, \phi\right)\right)+\mathcal{L}_{m}\right],
$$

where we set $8 \pi G=1$ and $\mathcal{L}^{m}$ is the Lagrangian for matter. The action above is obviously invariant under conformal transformation $\tilde{g}_{\mu \nu} \rightarrow \Omega^{2} \tilde{g}_{\mu \nu}$ because it depends only on $g_{\mu \nu}$ which is conformally invariant by itself. ${ }^{1}$

Variation of the action is given by

$$
\delta S=\int d^{4} x \frac{\delta S}{\delta g_{\alpha \beta}} \delta g_{\alpha \beta}=-\frac{1}{2} \int d^{4} x \sqrt{-g}\left(G^{\alpha \beta}-T^{\alpha \beta}\right) \delta g_{\alpha \beta},
$$

where $G^{\mu \nu}=R^{\mu \nu}-\frac{1}{2} R g^{\mu \nu}$ is the Einstein tensor and $T^{\mu \nu}$ is the energy momentum tensor for the matter. However, the variation $\delta g_{\alpha \beta}$ can be expressed in terms of the variation of the auxiliary metric $\delta \tilde{g}_{\alpha \beta}$ and $\delta \phi$, and takes the form

$$
\begin{aligned}
\delta g_{\alpha \beta} & =P \delta \tilde{g}_{\alpha \beta}+\tilde{g}_{\alpha \beta} \delta P \\
& =P \delta \tilde{g}_{\alpha \beta}+\tilde{g}_{\alpha \beta}\left(-\tilde{g}^{\kappa \mu} \tilde{g}^{\lambda \nu} \delta \tilde{g}_{\mu \nu} \partial_{\kappa} \phi \partial_{\lambda} \phi+2 \tilde{g}^{\kappa \lambda} \partial_{\kappa} \delta \phi \partial_{\lambda} \phi\right) \\
& =P \delta \tilde{g}_{\mu \nu}\left(\delta_{\alpha}^{\mu} \delta_{\beta}^{\nu}-g_{\alpha \beta} g^{\kappa \mu} g^{\lambda \nu} \partial_{\kappa} \phi \partial_{\lambda} \phi\right)+2 g_{\alpha \beta} g^{\kappa \lambda} \partial_{\kappa} \delta \phi \partial_{\lambda} \phi
\end{aligned}
$$

which implies that

$$
\begin{aligned}
\delta S= & -\frac{1}{2} \int d^{4} x \sqrt{-g}\left(G^{\alpha \beta}-T^{\alpha \beta}\right) \\
& \times\left(P \delta \tilde{g}_{\mu \nu}\left(\delta_{\alpha}^{\mu} \delta_{\beta}^{\nu}-g_{\alpha \beta} g^{\kappa \mu} g^{\lambda \nu} \partial_{\kappa} \phi \partial_{\lambda} \phi\right)+2 g_{\alpha \beta} g^{\kappa \lambda} \partial_{\kappa} \delta \phi \partial_{\lambda} \phi\right) .
\end{aligned}
$$

The corresponding equations of motion thus become

$$
\begin{aligned}
\left(G^{\mu \nu}-T^{\mu \nu}\right)-(G-T) g^{\mu \alpha} g^{\nu \beta} \partial_{\alpha} \phi \partial_{\beta} \phi & =0 \\
\frac{1}{\sqrt{-g}} \partial_{\kappa}\left(\sqrt{-g}(G-T) g^{\kappa \lambda} \partial_{\lambda} \phi\right)=\nabla_{\kappa}\left((G-T) \partial^{\kappa} \phi\right) & =0
\end{aligned}
$$

\footnotetext{
${ }^{1}$ We stress that our considerations are different from other works on tensor-scalar gravity such as disformal gravity which are extensions of Brans-Dicke type theories, where the scalar field is dynamical (See e.g. J. D. Bekenstein, Phys. Rev. D48 (1993) 3641 and for later developments and references therein M. Zumalacarregui and J. Garcia-Bellido, arXiv:1308.4685). In our case, and because of invariance under conformal transformations of the physical metric, we will show that the scalar field $\phi$ is equivalent to the scaling factor up to an integrating constant, and thus is not a new dynamical degree of freedom.
} 
where $\nabla_{\kappa}$ denotes the covariant derivative with respect to the metric $g_{\mu \nu}$. Notice that the auxiliary metric $\tilde{g}_{\mu \nu}$ does not appear in these equations by itself but only via the physical metric $g_{\mu \nu}$, while the scalar field $\phi$ enters the equations explicitly. As it follows from (1) that

$$
g^{\mu \nu}=\frac{1}{P} \tilde{g}^{\mu \nu},
$$

and therefore the scalar field satisfies the constraint equation

$$
g^{\mu \nu} \partial_{\mu} \phi \partial_{\nu} \phi=1
$$

Taking the trace of equations (6) we find that

$$
(G-T)\left(1-g^{\mu \nu} \partial_{\mu} \phi \partial_{\nu} \phi\right)=0
$$

and this equation is satisfied identically due to (8) even for $G-T \neq 0$. In fact the trace $G-T$ is determined by equations (7) and (8) and even in the absence of matter, when $T^{\mu \nu}=0$, the equations for the gravitational field have nontrivial solutions for the conformal mode. The field $\phi$ satisfies the Hamilton-Jacobi equation for a unit mass relativistic particle in a gravitational field (8) with the action identified with $\phi$ [1]. After solving it for $\phi$ equation (7) determines $G-T$. Thus the gravitational field, in addition to two transverse degrees of freedom, describing gravitons, acquires extra longitudinal degree of freedom shared by the scalar field $\phi$ and a conformal factor of the physical metric. This system however, is constrained by conformal invariance. To understand what this extra degree of freedom describes we rewrite equations (6) in the following form

$$
G^{\mu \nu}=T^{\mu \nu}+\tilde{T}^{\mu \nu}
$$

where

$$
\tilde{T}^{\mu \nu}=(G-T) g^{\mu \alpha} g^{\nu \beta} \partial_{\alpha} \phi \partial_{\beta} \phi,
$$

Now compare this expression with the energy momentum tensor for a perfect fluid

$$
T^{\mu \nu}=(\varepsilon+p) u^{\mu} u^{\nu}-p g^{\mu \nu},
$$

where $\varepsilon$ is the energy density, $p$ is the pressure and $u^{\mu}$ is four-velocity which satisfies the normalization condition $u^{\mu} u_{\mu}=1$. If we set $p=0$ and make the following identification

$$
\varepsilon \equiv G-T, \quad u^{\mu} \equiv g^{\mu \alpha} \partial_{\alpha} \phi,
$$

the energy momentum tensor (12) becomes equivalent to $\tilde{T}^{\mu \nu}$. Thus, the extra degree of freedom imitate the potential motions of "dust" with the energy density $G-T$ and the scalar field plays the role of the velocity potential. In the absence of matter this energy density is equal to $-R$, which does not vanish for generic solutions. As one can see the normalization condition for the four-velocity, $u^{\mu} u_{\mu}=1$, is equivalent to the scalar field equation (8) and the conservation law for $\tilde{T}^{\mu \nu}$ gives

$$
0=\nabla_{\mu} \tilde{T}_{\nu}^{\mu}=\partial_{\nu} \phi \nabla_{\mu}\left((G-T) \partial^{\mu} \phi\right)+(G-T) \partial^{\mu} \phi \nabla_{\mu} \partial_{\nu} \phi
$$


The second term here vanishes because by differentiating $g^{\mu \nu} \partial_{\mu} \phi \partial_{\nu} \phi=1$ we get $\partial^{\mu} \phi \nabla_{\nu} \partial_{\mu} \phi=0$ and $\nabla_{\nu} \partial_{\mu} \phi=\nabla_{\mu} \partial_{\nu} \phi$. Therefore the conservation law for $\tilde{T}^{\mu \nu}$ leads to equation (7).

To find the explicit solution of this equation it is convenient to work in synchronous coordinate system where the metric takes the form

$$
d s^{2}=d \tau^{2}-\gamma_{i j} d x^{i} d x^{j}
$$

with $\gamma_{i j}$ being a three dimensional metric. Moreover taking the hypersurfaces of constant time to be the same as the hypersurfaces of constant $\phi$ (see ([1]) for details), that is,

$$
\phi\left(x^{\mu}\right) \equiv \tau,
$$

we find that (8) is satisfied. In turn equation (7) becomes

$$
\partial_{0}(\sqrt{\operatorname{det} \gamma}(G-T))=0
$$

and hence

$$
G-T=\frac{C\left(x^{i}\right)}{\sqrt{\operatorname{det} \gamma}}
$$

where $C\left(x^{i}\right)$ is constant of integration depending only on spatial coordinates. In particular in flat Friedman universe, where

$$
\gamma_{i j}=a^{2}(\tau) \delta_{i j}
$$

we have

$$
G-T=\frac{C}{a^{3}},
$$

that is, we have a "dark matter" without dark matter, which is imitated by extra scalar degree of freedom of the gravitational field. With respect to the gravitational interaction this new mimetic dark matter behaves precisely in the same way as the usual dark matter (in particular, it is influenced by the gravitational instability), but it does not participate in any other interaction besides of the gravitational one. The "amount" of this mimetic dark matter is determined by the constant of integration $C\left(x^{i}\right)$.

To make the model above realistic in inflationary cosmology it must be modified. In fact, if inflation lasts longer than 70 e-folds, then for those initial conditions of $C\left(x^{i}\right)$ which do not spoil inflation, the "amount" of mimetic dark matter remaining at the end of inflation will be completely negligible. To protect its energy density from decay during the exponential expansion, $a=H^{-1} \exp (H t)$ one can, for example, introduce the coupling of field $\phi$ with the inflaton field $\varphi$ of the form

$$
\phi F(\varphi)
$$

where $F(\varphi)$ is some function of the inflaton field. In this case equation (17) is modified during inflation as

$$
\frac{1}{a^{3}} \partial_{0}\left(a^{3}(G-T)\right)=F(\varphi)
$$


Because the inflaton field is changing slowly the function $F(\varphi)$ can be taken as a constant and the approximate general solution of this equation is

$$
G-T \approx-\frac{F(\varphi)}{3 H}+C \exp (-3 H t)
$$

and at the end of inflation when the second term decays

$$
G-T \approx-\frac{F(\varphi)}{3 H}
$$

It is clear from here that if the inflaton field is slightly inhomogeneous then the produced mimetic dark matter will also be inhomogeneous and the resulting perturbations will be similar to adiabatic perturbations in case of real cold dark matter.

\section{Acknowledgments}

We thank Lars Brink and Costas Bachas for useful discussions. The work of AHC is supported in part by the National Science Foundation Phys-1202671 and by the Humboldt Foundation. The work of VM is supported by "Chaire Internationale de Recherche Blaise Pascal financée par l'Etat et la Région d'Ile-de-France, gérée par la Fondation de l'Ecole Normale Supérieure", by TRR 33 "The Dark Universe" and the Cluster of Excellence EXC 153 "Origin and Structure of the Universe".

Open Access. This article is distributed under the terms of the Creative Commons Attribution License which permits any use, distribution and reproduction in any medium, provided the original author(s) and source are credited.

\section{References}

[1] L. Landau and E. Lifshitz, The Classical Theory of Fields, section 97, fourth edition, Butterworth, Heinemann (1980). 